\title{
Suitably qualified 8 persons
}

\section{KEY POINTS}

- Suitability qualified persons (SQPS) can prescribe and supply 'prescription-only medicines - veterinarian, pharmacist, SQP' (POM-VPS) and 'non-food animal medicines - veterinarian, pharmacist, SQP' (NFA-VPS) medicines

- SQPs must operate from an approved premises

- SQPs cannot diagnose disease

- SQPs must assess owners' competence and advise on warnings and safe administration

- SQPs cannot prescribe 'prescription-only medicine - veterinarian' (POM-V) medicines or initiate use of the cascade

\section{Definitions}

'Suitably qualified person (SQP)' is a phrase used in the Veterinary Medicines Regulations (VMR) to describe a person who is permitted to prescribe and supply veterinary medicines classified as POM-VPS or NFA-VPS in the UK. Most of the medicines in the 'veterinarian, pharmacist, SQP' (VPS) categories have preventive uses (e.g. external and internal antiparasitic medicines, farm animal vaccines and nutritional supplements). SQPs may, like anyone else, supply medicines classified AVM-GSL and those sold under the exemption scheme for small pet animals. SQPs must register with the Animal Medicines Training Regulatory Authority (AMTRA), Vetpol or VetSkill. It should be noted that holding an RVN (registered veterinary nurse) qualification does not allow the person to supply NFA-VPS products unless they also hold an SQP qualification.

Over 7000 SQPs are registered with AMTRA. Such SQPs have to renew their registration with AMTRA each year and pay an annual fee. AMTRA monitors continuing professional development (CPD) and deals with complaints about breaches of professional standards.
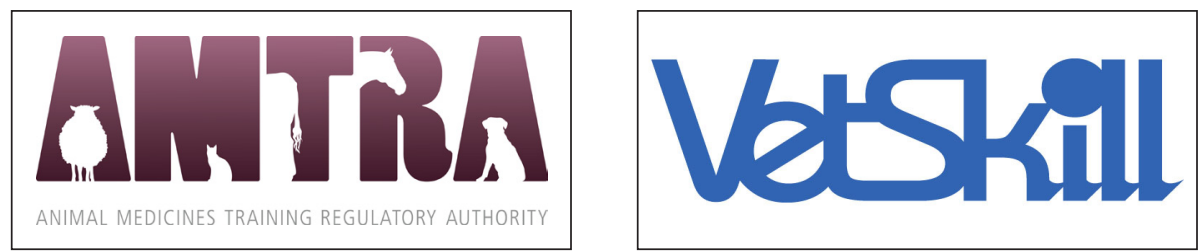

SQPs must comply with a Code of Practice issued by the Department for Environment, Food and Rural Affairs (Defra) Secretary of State through the Veterinary Medicines Directorate (VMD) and distributed by AMTRA. SQPs must supply only from authorized premises and only from within the animal group categories for which they are trained and registered.

SQP should not be confused with the broader term Registered Qualified Person (RQP) which encompasses:

- Veterinary surgeons registered with the Royal College of Veterinary Surgeons (RCVS)

- Pharmacists registered with the General Pharmaceutical Council (in Great Britain) or the Pharmaceutical Society of Northern Ireland

- SQPs registered with AMTRA or VetSkill.

The regulations also define other types of 'qualified person', including Manufacturing Qualified Persons and Pharmacovigilance Qualified Persons.

The category of an SQP is indicated by a character or characters within their SQP number. The most common are:

- R-SQP - qualified to supply for all species groups

- E-SQP - equine and companion animal only

- C-SQP - companion animal only.

There are other potential species combinations with their own prefixes. More information can be found on the AMTRA website (@)) 


\section{Premises}

SQPs must operate from approved premises, which may be a registered veterinary practice or a registered pharmacy, where no further registration is needed, or an SQP retailer's premises registered with the VMD. The VMD will inspect premises and register them annually. For more information, see the VMD's guidance on retail of veterinary medicines (ㅇ) .

\section{See also Registration of premises and inspections.}

\section{Legal duties}

When prescribing a POM-VPS product, the SQP must always take account of:

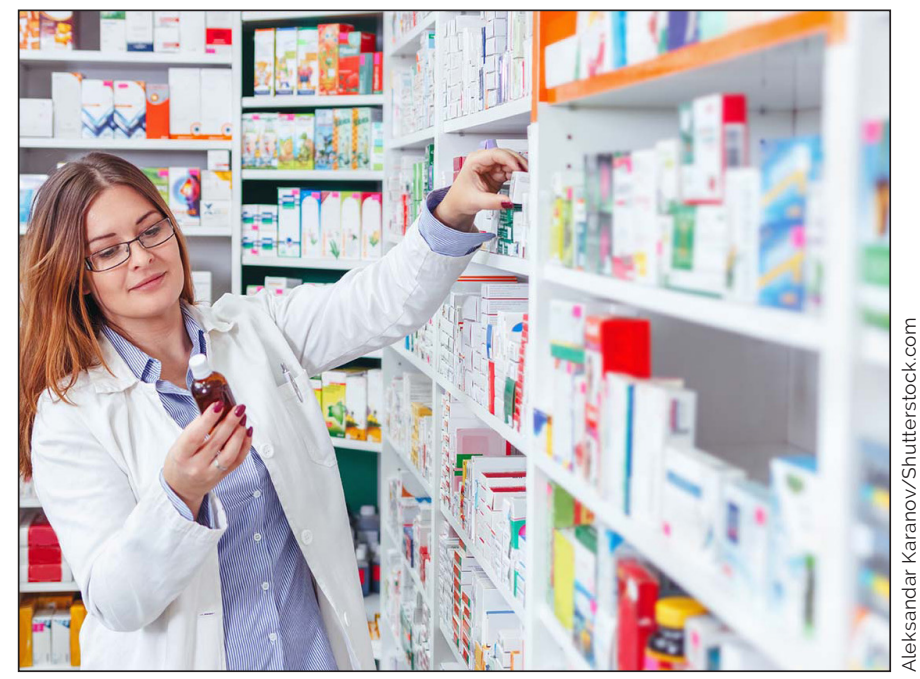

- The disease/condition of the animals requiring treatment

- The type of holding and the animals being treated

- The authorized veterinary medicines on the market, and their warnings and contraindications

- The responsible use of medicines (see below)

- The requirement to prescribe the minimum amount of product necessary for the treatment and condition presented

- The requirement for the person receiving the product to use it for an authorized use

- The abilities and competence of the person who will administer the product

- Any available farm or animal health plan.

That prescription may be in writing but usually will be verbal. SQPs need not see the animal and in any case may not diagnose disease.

For horses and other equidae, the SQP must check whether the animal has been declared as non-food-producing in their horse passport.

For anthelmintic products for sheep and cattle, SQPs should follow the recommendations of the Sustainable Control of Parasites in Sheep (SCOPS) and the Control of Worms Sustainably (COWS) groups.

In supplying a POM-VPS or NFA-VPS product, the SQP must always:

- Be satisfied that the person who will use the product is competent to use it safely and for its authorized use

- Advise on warnings or contraindications

- Provide advice on safe administration.

SQPs may not break the immediate packaging of a medicine, so cannot supply a small number of tablets from a tub, but may for instance supply individually-wrapped boluses or parts of a blister strip of tablets, provided that all the required written information is supplied to each client, such as by providing a copy of the package insert or summary of product characteristics (SPC).

\section{Cascade use}

On their own authority, an SQP may only supply medicines and advise on use consistent with the SPC. If a client wishes to use a medicine for a species for which it is not authorized, or by an administration route not authorized, or under a different dosage regime, then an SQP may only supply the medicine in accordance with a prescription from a veterinary surgeon under the prescribing cascade. The prescription should be retained for inspection.

See also Prescribing cascade.

\section{Examinations and categories}

The Code of Practice for SQPs requires that all SQP qualifications be at Higher Education Level 4 (equivalent to first-year degree level) or above, unless integrated within a broader veterinary nursing qualification (when they may be at level 3) and outlines the required syllabus.

Candidates seeking to become AMTRA SQPs are assessed by examinations set and marked by Harper Adams University taking place at locations throughout the UK. All AMTRA SQPs have to pass an AMTRA viva and relevant written examinations. Additional species modules may be added at future dates, extending the range of medicine groups available to the SQP.

The most common route to AMTRA SQP qualification is to pass a base examination, which covers legislation, anatomy, physiology and disease challenges. In addition to the base module, there are species modules: farm animal, equine, avian and companion animal. SQPs have to pass relevant species group module(s), as well as the base and oral examinations. Thus, SQPS can combine species modules to create the qualification relevant to them and their business

Alternatively, qualified registered veterinary nurses may become SQPs via a shorter written examination, which concentrates on legislation and application of the knowledge and understanding they are already likely to have, in order to become a C-SQP. They can build on this by adding the farm animal, avian or equine modules.

Some veterinary pharmacy qualifications are also recognized by AMTRA as the academic basis for SQP registration. 


\section{Continuing professional development}

Once qualified, an AMTRA SQP must show they are keeping up to date. There is a two-yearly requirement for CPD points. These can be gained from accredited meetings and webinars, accredited distance learning and private study. CPD is compulsory: those not gaining enough CPD points cannot continue as SQPs without passing fresh examinations. More information can be found on the AMTRA CPD webpage (@)).

\section{SQPs in veterinary practice}

An SQP working in a veterinary practice has the legal right to prescribe and supply POM-VPS and NFA-VPS medicines without recourse to the veterinary surgeon, and to anyone, not just clients of the practice. Without an SQP, every decision to supply any medicine (other than those on free sale) must be made by a veterinary surgeon on a case-by-case basis, which may pose logistical challenges as well as potentially inhibiting clients and thus compromising animal care.

Being an SQP gives no extra rights in relation to POM-V medicines. The veterinary surgeon must prescribe the product and authorize each transaction individually, but may authorize another person to hand over the product provided the veterinary surgeon is satisfied that the person handing it over is competent to do so. It is not necessary to be an SQP to be regarded as competent.

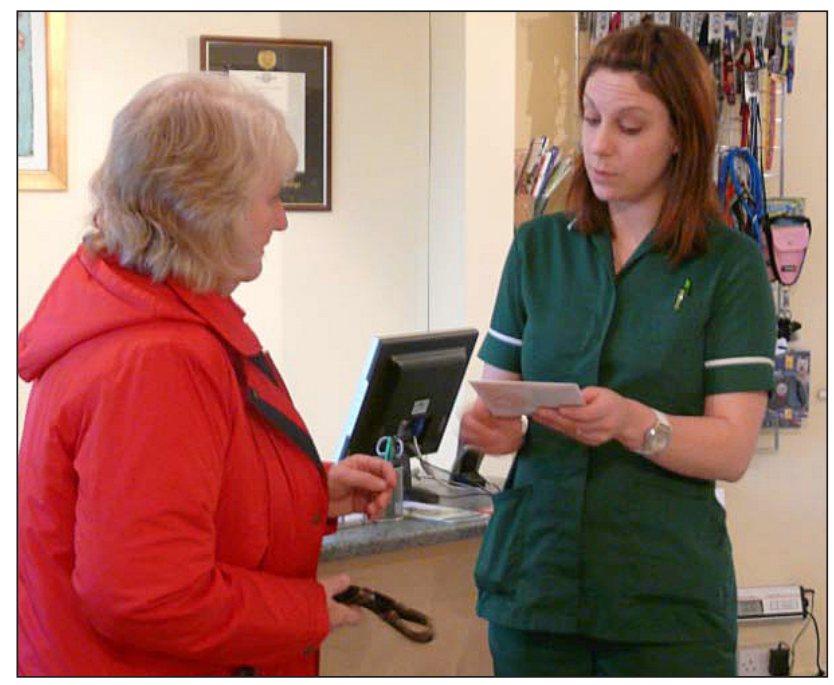

\section{QUESTIONS}

1. Which organization is responsible for registering SQPS?
a. AMTRA or VetSkill
b. VMD
c. $\mathrm{NOAH}$
d. The Home Office

2. Which categories of medicines can SQPs supply or prescribe?
a. POM-V; POM-VPS
b. POM-VPS; NFA-VPS; AVM-GSL
c. POM-V; NFA-VPS
d. POM-V; POM-VPS; NFA-VPS; AVM-GSL

3. When supplying a POM-VPS or NFA-VPS medicine, an SQP must:
a. Be satisfied that the person who will use the product is competent to use it safely
b. Advise on warnings or contraindications
c. Provide advice on safe administration
d. All of the above

4. SQPS working in veterinary practice can:
a. Prescribe POM-V medicines
b. Use the prescribing cascade
c. Prescribe and supply POM-VPS and NFA-VPS medicines without recourse to the veterinary surgeon
d. Supply all wormers and flea treatments to non-clients 